\title{
1 On-line FTIR as a novel tool to monitor Fenton process behavior
}

2 Noemí Merayo, Daphne Hermosilla ${ }^{*}$, Carlos Negro and Ángeles Blanco

3

4 Department of Chemical Engineering, Complutense University of Madrid, Facultad de Ciencias

5 Químicas, Ciudad Universitaria s/n, 28040 Madrid, Spain.

6

7

$8 \quad{ }^{*}$ Corresponding author. Tel.: +34 91394 4645; fax: +34 913944243.

9 E-mail address: dhermosilla@quim.ucm.es (D. Hermosilla). 


\section{ABSTRACT}

2 The efficiency of advanced oxidation processes is usually optimized by measuring the

3 evolution of some water quality parameters sampling aliquots at pre-selected time

4 intervals, such as particular undesired contaminants contents, or the reduction of

5 chemical oxygen demand and total organic carbon. Besides providing good information

6 regarding overall treatment performance and dynamics, this methodology also implies

7 large analytical time consumption, and does not offer the actual full sequence of

8 compounds appearing and disappearing during oxidation. On-line Fourier transform

9 infrared spectroscopy is herein reported as a very useful tool for this purpose. In

10 particular, it was successfully applied to monitoring the Fenton's oxidation of three

11 model compounds (phenol, acetic acid, and oxalic acid) performed in continuous,

12 providing precise control of the effect of reagents over time. Hydroxylation reactions

13 resulted in the formation of hydroquinone and catechol as the main aromatic by-

14 products being generated along the oxidation of phenol by the Fenton process. All

15 phenolic substances (phenol, hydroquinone, benzoquinone, and catechol) were totally

16 removed along the reaction. Carboxylic acids (oxalic and acetic mainly) were

17 significantly present as final by-products of the oxidation process, highlighting their

18 oxirecalcitrant behavior. On-line FTIR successfully enabled monitoring the Fenton

19 process, and it provided a precise control of the effect of reagents along reaction time.

20 Applications for a future on-line control of Fenton processes in industry may be

21 developed in order to optimize the use of reagents and the potential combination with

22 biological treatment stages; therefore reducing the operational cost of this advanced

23 oxidation treatment.

24 Keywords: Advanced oxidation processes; Fenton method; Fourier transform infrared

25 spectroscopy; treatment on-line monitoring; phenol. 


\section{1. Introduction}

2 Advanced oxidation processes (AOPs) involving the in situ generation of highly

3 reactive transitory species (e.g. $\left.\mathrm{H}_{2} \mathrm{O}_{2}, \mathrm{OH} \cdot, \mathrm{O}_{3}, \mathrm{O}_{2}^{-} \cdot\right)$ are taking advantage when

4 conventional wastewater treatment techniques become insufficient to treat biorefractory

5 contaminants [1-5]. Particularly, the method described by Fenton [6] is one of the most

6 frequently used because it is generally more efficient, and implies a significant lower

7 economical cost than other AOPs $[2,5,7,8]$.

The Fenton process is based on the electron transfer between hydrogen peroxide

$9 \quad\left(\mathrm{H}_{2} \mathrm{O}_{2}\right)$ and ferrous ion $\left(\mathrm{Fe}^{2+}\right)$, which acts as a homogenous catalyst, generating

10 hydroxyl radicals $\left(\mathrm{OH}^{-}\right)$that can degrade organic compounds [9]. These highly reactive

11 radicals initiate the oxidative destruction of organic substances $(\mathrm{RH})$ present in

12 wastewater by hydroxyl radical addition or hydrogen atom abstraction reactions [5].

13 Organic free radicals $\left(\mathrm{R}^{*}\right)$ are formed as transient intermediates that are further oxidized

14 by hydroxyl radical, hydrogen peroxide, oxygen, ferric iron, and other oxidative

15 intermediates; finally yielding stable oxidized products [5].

16 The optimization and process control of Fenton treatment and other AOPs has

17 usually been undertaken by measuring certain water quality parameters (e.g. undesired

18 contaminants contents, chemical oxygen demand, and/or total organic carbon removal)

19 at certain time intervals. In addition, several techniques have widely been applied to

20 characterize the sequence of organic compounds that are produced during the oxidative

21 treatment using this discrete sampling protocol, such as high-performance liquid

22 chromatography (HPLC) [10-12], gas chromatography-mass spectrometry (GC-MS)

23 [13-14], Fourier transform infrared spectroscopy (FTIR) [15-16], or different

24 combinations of them, or with other analytic techniques (e.g. ultraviolet-visible 
1 spectrophotometry (UV-Vis), liquid chromatography-mass spectrometry (LC-MS), ion

2 chromatography (IC), etc.) [17-21].

Particularly, FTIR has been previously applied to analyze the surface of catalysts and adsorbed substances along diverse AOPs treatments [22-25]. Gas samples have also been analyzed by FTIR to measure the generation of carbon dioxide (assimilable as

6 mineralized carbon) in outlet gaseous streams of ozonation processes, which served as

7 an indirect control parameter of the treatment [26]. In addition, FTIR has also been

8 applied to control reactions in liquid samples identifying the compounds that are

9 appearing and disappearing in the solution at preset time intervals [15-16]. All these

10 methods enabling an indirect discrete control of the reaction involve great analytical

11 time investment, and do not provide a full continuous characterization of the sequence

12 of compounds that are produced in, or removed from the solution along the process.

13 Furthermore, no reference for its on-line application has been reported to date.

On the other hand, membrane-introduction mass spectrometry (MIMS) has actually been applied to perform on-line measurements along photocatalytic processes

16 [27]. Nevertheless, despite this methodology has been reported useful for monitoring

17 these processes on-line, just volatile organic pollutants that are present in water can be effectively analyzed; whereas other highly polar substances could not be detected

19 properly, such as some compounds that have been previously reported to be typically 20 generated along the oxidation process of phenol [27]. Moreover, the current tendency of improving the combination of AOPs with 22 biological technologies [28, 29] would surely welcome the application of advanced analytical methods to optimize the efficiency of every treatment step considering the

24 predominant bio- or oxi- degradable nature of by-products. Therefore, the main 25 objective of this essay was developing a suitable methodology of on-line monitoring the 
1 evolution of the Fenton treatment of model organic compounds based on FTIR; which

2 may ultimately allow the addition of reagents to be optimized, a further identification of

3 the involved reactions, and the qualitative and quantitative determination of the by-

4 products that are generated along the process.

6 2. Material and methods

$7 \quad$ 2.1. Material and analytical methods

8 All used chemicals were of analytical grade and supplied by PANREAC S.A.

9 (Barcelona, Spain) and Sigma-Aldrich (Highland, USA). Solutions were prepared in

10 ultrapure water and kept in dark until use. $0.1 \mathrm{~N} \mathrm{H}_{2} \mathrm{SO}_{4}$ and $0.1 \mathrm{~N} \mathrm{NaOH}$ were used to

11 adjust the $\mathrm{pH}$ value of the solution along the process.

12 The concentration of each tested organic compound (16 mmol of phenol, 14

$13 \mathrm{mmol}$ of oxalic acid, and $12 \mathrm{mmol}$ of acetic acid in a total volume of $100 \mathrm{~mL}$ ) was

14 considered in order to achieve a good monitoring resolution of the process in the

15 ReactIR iC10 device. Phenol was chosen as a model compound to perform this essay

16 because its degradation behavior by several AOPs (Fenton process included) has widely

17 been described previously [30-34], so it would perfectly serve to evaluate the proposed

18 methodology. In addition, oxalic and acetic acids were also chosen due to its

19 oxirecalcitrant nature [32].

All analyses were made according to the standard methods for the examination

21 of water and wastewaters [35]. Chemical oxygen demand (COD) was measured by the

22 colorimetric method at $600 \mathrm{~nm}$ using an Aquamate-spectrophotometer (Thermos

23 Scientific AQA 091801, Waltham, USA); and hydrogen peroxide concentration was

24 analyzed using the titanium-sulphate spectrophotometric method [36]. As residual

25 hydrogen peroxide in the solution interferes with COD analysis, this interference was 
1 corrected fitting the relationship between COD and hydrogen peroxide content to a

2 second order polynomial equation $\left(\mathrm{DQO}\left(\mathrm{H}_{2} \mathrm{O}_{2}\right)=-0.000020 \cdot\left[\mathrm{H}_{2} \mathrm{O}_{2}\right]^{2}+\right.$

$\left.3 \quad 0.393239 \cdot\left[\mathrm{H}_{2} \mathrm{O}_{2}\right] ; \mathrm{R}^{2}=99.92 \% ; \mathrm{p}=0.0001\right)[37]$.

4

Total organic carbon (TOC) was measured by the combustion-infrared method using a TOC/TN analyzer multi N/C $\mathrm{C}^{\circledR} 3100$ (Analytik Jena AG, Jena, Germany) with catalytic oxidation on cerium oxide at $850^{\circ} \mathrm{C}$. The integration of the information provided by the evolution of both COD and TOC along the oxidation treatment was assessed by calculating the mean oxidation number of organic carbon $(\mathrm{MOC}=4 \cdot[1-$ (COD/TOC)], considering both COD and TOC in molar units)[32].

Phenol and reaction intermediates were complementary measured by High Pressure Liquid Chromatography (Model L920, Varian, CA, USA) with diode array (PDA) detection. Acetonitrile - water (15\%:85\%), and (50\%:50\%) were used as the eluent for aromatics and carboxylic acids, respectively. Sample injections of $20 \mu \mathrm{L}$ were separated on a C-18 column (Vidac $250 \mathrm{~mm} \times 4.6 \mathrm{~mm}$ ID $\times 5 \mu \mathrm{m}$ ) at $30^{\circ} \mathrm{C}$. The target compounds were measured at the following wavelengths: hydroquinone $(290 \mathrm{~nm})$, benzoquinone $(245 \mathrm{~nm})$, catechol $(280 \mathrm{~nm})$, phenol $(270 \mathrm{~nm})$, acetic acid and oxalic acid $(200 \mathrm{~nm})$.

\subsection{FTIR analytical device}

ReactIR iC10 (Mettler-Toledo, Columbia, USA) is a real-time in situ reaction monitoring system, based on FTIR spectrometry, that is able to provide all the organic chemical species that are present in the solution as the reaction is being performed. The FTIR spectrometer uses a mercury-cadmium telluride (MCT) detector that is cooled by liquid nitrogen; and measurements are optically taken using a diamond-tipped probe with a 1 meter long fibre-optic conduit. This system was purged using instrumental- 
1 grade air; therefore preventing water vapour from collecting inside the optics, which

2 may obscure spectral data otherwise.

3 Data acquisition was performed from 2000 to $650 \mathrm{~cm}^{-1}$ with an $8 \mathrm{~cm}^{-1}$ nominal

4 resolution. 256 scans were co-added for each spectrum. A background of pure water

5 was carried out using the same resolution and scanning conditions of the trials before

6 each spectral record. These water spectra were subtracted from each corresponding

7 resulting on-line spectra.

Real-time component analyses were performed using ConcIRT software

9 (Mettler-Toledo, Columbia, USA), which applies the curve-resolution mathematical

10 algorithm for grouping wavenumber values that change absorbance intensity in the

11 same way. This software calculates the associated component spectrum and the relative

12 concentration profile in terms of absorbance units for each group; and it re-analyzes and

13 updates all spectra and concentration profiles as each new reaction spectrum is acquired.

14 In short, calculation results evolve as the reaction proceeds, and every organic

15 component (reagent, intermediate or product) that is present in the solution is detected

16 in real-time; and its relative concentration profile is therefore provided.

\subsection{Experimental procedure}

19 Experiments were performed in the dark inside a $500 \mathrm{~mL}$ glass reactor where

$20100 \mathrm{~mL}$ of the solution were continuously stirred (300rpm) with a magnetic device at

21 room temperature $\left(\approx 20-25^{\circ} \mathrm{C}\right)$. All experiments were repeated three times. $\mathrm{pH}=2.8( \pm$

22 0.2) was kept constant along the process. $\mathrm{pH}$ adjustment was required until this 2.8

23 value was monitored stable and no further regulation was necessary. The concentrations

24 of reagents were chosen to meet the following ratios: $\left[\mathrm{H}_{2} \mathrm{O}_{2}\right] / \mathrm{COD}_{0}=2.15$, and $\left[\mathrm{H}_{2} \mathrm{O}_{2}\right]$ 
$1 \quad /\left[\mathrm{Fe}^{2+}\right]=37.5$; as these reaction conditions have previously been reported to produce

2 optimal Fenton treatment results for the target substances [30, 32]. The required amount of ferrous sulphate was added in batch mode after the

$4 \quad$ initial $\mathrm{pH}$ value was adjusted. Hydrogen peroxide was thereafter added in continuous

5 mode, so all the designed dosage of this reagent was supplied after 120 minutes of

6 reaction. In fact, the continuous addition of $\mathrm{H}_{2} \mathrm{O}_{2}$ has already been reported to provide

7 better results than batch mode in previous trials of this process [31, 37]. In addition, it

8 also allows a better understanding of the process enabling the use of the added quantity

9 of $\mathrm{H}_{2} \mathrm{O}_{2}$ (milimol), rather than reaction time, as an indicator of the progress and

10 evolution of oxidation.

11 Additional trials were performed in equal conditions in which aliquots of the

12 solution were withdrawn with a syringe in order to monitor the overall progress of the

13 reaction in terms of COD removal and $\mathrm{H}_{2} \mathrm{O}_{2}$ consumption. This way, volume changes

14 did not alter FTIR-monitoring runs. These samples were neutralized to $\mathrm{pH}=9.0$ with

$1540 \% \mathrm{NaOH}$, mixed, and centrifuged for $15 \mathrm{~min}$ at $2000 \mathrm{rpm}$, before collecting the

16 supernatant where COD and $\left[\mathrm{H}_{2} \mathrm{O}_{2}\right]$ were determined.

17 The ReactIR iC10 probe placed inside the reactor monitored the whole reaction

18 progress in real time. In order to properly read and calibrate spectral results, the

19 characteristic bands of the main compounds that were expected to be produced during

20 the Fenton treatment of the target substances were crosschecked using the available

21 information found in bibliography (Table A.1), and our own collected spectra from

22 conveniently prepared control solutions containing just one of these compounds

23 (Figures A.1 and A.2), which have been included in Appendix A. Despite the similarity

24 of some by-products of the reaction, the full interpretation of the generated complex 
1 spectra recorded during the trials was successfully achieved thanks to the mathematical

2 algorithms provided by ConcIRT software.

3

\section{$4 \quad 3$. Results and discussion}

$5 \quad$ 3.1. Degradation of phenol by the Fenton process

6 All three repetitions of every performed experiment reproduced the same results, and

7 there were not found meaningful differences among them.

Spectra within the region of 650 to $2000 \mathrm{~cm}^{-1}$ continuously increased during

$9 \mathrm{H}_{2} \mathrm{O}_{2}$ addition until $60 \mathrm{mmol}$ of $\mathrm{H}_{2} \mathrm{O}_{2}$ were added in total, when the highest overall

10 absorbance was reached. Subsequently thereafter, the overall spectra began to decrease

11 and all peaks progressively became smoother (Figure 1).

After the first addition of $\mathrm{H}_{2} \mathrm{O}_{2}$, the color of the solution instantaneously changed from colorless to dark brown (almost black) due to the oxidation of ferrous to

14 ferric ion, and the generation of quinones (i.e. hydroquinone and benzoquinone, Figure

15 2) in redox equilibrium [31,33], which are more toxic than phenol itself [31]. This dark

16 color gradually lost its intensity thereafter, and a pale orange color remained after

17 adding a total $160 \mathrm{mmol}$ of $\mathrm{H}_{2} \mathrm{O}_{2}$ (when its ratio to the initial amount of phenol was 10 )

18 because quinones have already been degraded; as well as due to the remaining presence

19 of several by-products of acid formation stages (oxalic acid, mainly) [32-33] that are

20 able to reduce ferric back to ferrous iron [38]. Similar color changes have been

21 addressed before [33, 39]. Furthermore, HPLC analyses confirmed these FTIR results.

22 The evolution of MOC along treatment (Figure 2) also supports this affirmation

23 linearly changing from an initial value of -0.67 , which is characteristic for phenol, to $\approx 3$,

24 which has previously been addressed to a mix of carboxylic acids where oxalic (MOC =

253 ) predominates [32]; and then it remained constant thereafter. 
2 phenol itself and some products that are generated by its hydroxylation, namely

3 hydroquinone, benzoquinone [31, 33, 40] and catechol (Figure 2). On the other hand,

4 resorcinol was not actually found along the Fenton oxidation of phenol. In fact, its

5 formation would rather be implausible based upon the substitution rules of organic

6 chemistry [33]; and it may anyhow occur in an about one thousand times lower

7 frequency than the generation of catechol and hydroquinone [34].

The absorbance concentration profile of phenolic compounds logically increased

9 as phenol was added to the solution; and it also grew again after the addition of $\mathrm{H}_{2} \mathrm{O}_{2}$

10 because other phenolic intermediate compounds of the reaction were newly formed

11 (Figure 2). During the reaction, this phenolic mix totally disappeared when the

12 concentration ratio between the added $\mathrm{H}_{2} \mathrm{O}_{2}$ and the initially supplied amount of phenol

13 was close to 8, coinciding with previously reported results on the Fenton oxidation of

14 phenol and nitrophenol, when a MOC value characteristic of the predominant presence

15 of carboxylic acids $(\approx 2.5-3)$ was kept more or less constant as the reaction progresses

16 (Figure 2) [32].

Achieving the total degradation of hydroquinone is of environmental concern due to its high toxicity, which is several orders of magnitude higher than the attributed to phenol itself [29]. On the other hand, catechol also resulted totally removed at the end of the reaction, as it has been clearly addressed by HPLC measurements. Nevertheless, 21 catechol is highly biodegradable [41, 42]; thus, it might be further treated by biological technologies, which are, in general, cheaper treatments than AOPs. Furthermore, previous results reporting a significant much higher production of catechol than hydroquinone along the process were confirmed [33-34], as it results from comparing the concentration profiles of both compounds in Figure 2. In fact, the 
1 production of catechol resulted a $100 \%$ higher than the measured for quinones by

2 HPLC. In short, catechol and hydroquinone were initially formed as phenol

3 disappeared; and then, they began to be gradually degraded competing with their own

4 further formation as phenol was still being oxidized.

In addition, phenol decreased its concentration in the solution faster than the

6 other newly generated phenolic intermediates (hydroquinone, benzoquinone, and

7 catechol) of the reaction (comparing Figures 2 and 3); which is also in accordance to

8 previous scientific reports [32-34]. Only a 4\% of phenol remained after adding a ratio of

$9 \quad \mathrm{H}_{2} \mathrm{O}_{2}$ to phenol of 3.9; results that were further confirmed by HPLC analyses.

At this point, the removal of COD was higher than 50\%, and it did not show

11 further lineal progress (Figure 3). Finally, phenol resulted totally degraded when the aggregated concentration of $\mathrm{H}_{2} \mathrm{O}_{2}$ reached 5.6 times the initial amount of added phenol, and the reduction of the COD was close to a $75 \%$; showing a further asymptotic evolution because of the growing presence of carboxylic acids, which are more difficult to oxidize. Hereafter, the mix of phenolic compounds remaining in the solution was mainly made up by hydroquinone and catechol (Figure 2), which absorbance concentration profile quickly decreased until the ratio between the total added $\mathrm{H}_{2} \mathrm{O}_{2}$ and the initial amount of phenol was close to 6.5. Then, its abatement thereafter progressed smooth, and its total degradation was finally achieved when this ratio arrived to about 8 , as confirmed by HPLC determinations.

Some carboxylic acids remained in the solution at the end of the process as the main persistent by-products of the oxidation treatment of phenol (Figure 1); although they are also considered highly biodegradable and might be further treated by biological

24 processes [41, 42]. Oxalic, acetic, and formic acids were identified as the resultant products of an acid formation stage within the process. Its presence and persistence was 
1 also confirmed by HPLC measurement, and MOC and COD behavior assessment

2 (Figures 2 and 3), as just stated before. That is, its presence resulted constant after the

3 ratio of total added $\mathrm{H}_{2} \mathrm{O}_{2}$ to the initial amount of phenol reached a value close to 8 ,

4 which was accurately measured by HPLC; and also confirmed by a non further change

5 of MOC from characteristic values previously addressed for carboxylic acids mix [32],

6 although COD was still slightly being removed (Figures 2 and 3).

7 In short, carboxylic acids were probably formed by ring-opening reactions that

8 take place within degradation stages of some aromatic intermediate products of the

9 reaction [32-33]. Whilst the presence of oxalic acid was detected from almost the

10 beginning of the reaction (Figure 2), when the ratio of total added $\left[\mathrm{H}_{2} \mathrm{O}_{2}\right]$ to the initial

11 concentration of phenol was just 1.8; acetic and formic acids presence was noticed in

12 the solution when this ratio reached 6.25, suggesting that these two carboxylic acids

13 may be generated by the degradation of some other intermediate reaction by-products.

14 All these carboxylic acids that are inevitably formed during the oxidative

15 degradation of phenol are more or less recalcitrant to its further Fenton advanced

16 oxidation treatment $[1,16]$; so they will hereafter be considered as oxirecalcitrant

17 compounds [32]. In fact, this limited capacity to degrade carboxylic acids is one of the

18 main drawbacks for achieving the total mineralization of phenol by Fenton's reagent

$19[32,33]$. Therefore, the main objective of AOPs based treatment steps might be defined

20 as controlling the process until a maximum biodegradability threshold is achieved in

21 order to combine this treatment with a cheaper posterior biological stage [43].

22 In summary, a final 94\% reduction of the COD was achieved (Figure 3), which

23 is even higher than previously reported results [30,32]. The continuous addition of

$24 \mathrm{H}_{2} \mathrm{O}_{2}$, which has previously been proved to enhance the removal of COD in comparison

25 to batch mode [37], as well as the steady thorough control of the reaction conditions that 
1 was performed, have surely served well to achieve this very successful result. The $6 \%$

2 remaining COD was held by the remaining mix of oxirecalcitrant by-products, oxalic

3 and other carboxylic acids, mainly.

4

5

6

$7 \mathrm{FeSO}_{4} \cdot 7 \mathrm{H}_{2} \mathrm{O}$ resulted in a colorless solution because the high production of a complex

8 that is formed between acetic acid and ferrous iron drastically reduced the presence of

9 both compounds in the solution (Figure 4). $\mathrm{As}_{2} \mathrm{O}_{2}$ was thereafter added, the

10 concentration of this complex began to decrease, and the solution turned to an orange-

11 reddish color as ferric ion was generated [38], which further induced the formation of an

12 acetic acid-ferric iron complex. The formation of this complex is stronger than the

13 acetic acid-ferrous iron one [32], but it was not detected by FTIR in the spectral region

14 under study (Figure 5). Nevertheless, the clearly noticed orange color of the solution,

15 and the very limited figures of COD reduction, clearly suggest the presence of this

16 ferric-acetic acid complex, as it has previously been reported [32].

The reaction may be considered finished when the ratio between the added concentration of $\mathrm{H}_{2} \mathrm{O}_{2}$ and the initial amount of supplied acetic acid reached 4.8. At this moment, the organic-ferrous iron complex disappeared because there was not any available $\mathrm{Fe}^{2+}$ to form more $\mathrm{OH} \cdot$ that might have further continued the oxidation process, and the acetic acid-ferric iron complex (not visible to FTIR) and formic acid (Figure 5) remained in the solution as the final result of the attempted degradation of acetic acid by the Fenton's reagent [44-45]. Final COD reduction figures were just about a $9 \%$ due to the above mentioned oxirecalcitrant nature of this type of chemicals. In fact, these poor treatment results fully agree with other previously reported ones [32]. 


\section{3.3. Fenton oxidation of oxalic acid}

A constant weak green-yellow color predominated in the solution along the

4 treatment of oxalic acid by Fenton's reagent; even after $\mathrm{H}_{2} \mathrm{O}_{2}$ was added. Therefore,

5 ferrous to ferric ion oxidation was occurring at a very low pace; being oxalic acid itself

6 contributing to reduce ferric back to ferrous [38]. This process partially slowed the

7 oxidative process down because some $\mathrm{H}_{2} \mathrm{O}_{2}$ was being wasted on oxidizing ferrous iron

8 back to ferric one.

In short, Fenton oxidation did not produce any degradation of oxalic acid (Figure

10 6). In fact, great $\mathrm{Fe}^{2+}$ losses have previously been attributed to the formation of a strong

11 oxalic-ferrous complex, which consequently hinders the oxidation process to progress

12 [32]. Although this oxalic-ferrous complex was not detected by the FTIR probe,

13 probably due to its very close likeness to other oxalic compounds, the oxidation process

14 resulted similarly hindered.

15 In fact, the absorbance concentration profile of oxalic acid did not show any

16 change after increasing the addition of $\mathrm{H}_{2} \mathrm{O}_{2}$ (Figure 6); although it significantly

17 decreased previously, just after adjusting the $\mathrm{pH}$ (adding $40 \% \mathrm{NaOH}$ ), because oxalic

18 acid and oxalate anion contents depend on the $\mathrm{pH}$ value of the solution [46]. That is,

19 oxalate anion was formed as $\mathrm{pH}$ increased, as it is detailed in Figure 7. Whereas the

20 characteristic wavenumber peaks of oxalic acid (1733 and $\left.1232 \mathrm{~cm}^{-1}\right)$ gradually

21 decreased during the addition of $40 \% \mathrm{NaOH}$ until its content stabilized at a very

22 constant final value [46]; those typical peaks of oxalic anion $\left(1571\right.$ and $\left.1310 \mathrm{~cm}^{-1}\right)$

23 showed up, and correspondingly increased its absorbance record, after $\mathrm{NaOH}$ was

24 added, until its content reached a steady state as well [46]. 
Summing up, the total removal of the COD that was achieved in the treatment of

2 oxalic acid by Fenton's reagent resulted lower than the 7\%. This result totally meets

3 previously reported results [31-32], which also suggested that it is the variation of $\mathrm{pH}$,

4 rather than the oxidative treatment itself, which is the responsible of this reduction of

5 the COD. In fact, this slight percentage of COD removal was surely the result of the

6 final precipitation of oxalic acid when $\mathrm{pH}$ was turned to 9 adding $\mathrm{NaOH}$ at the end of

7 the reaction aiming to remove iron precipitating its hydroxides.

\section{4. Conclusions}

10 The above reported results clearly show that the effectiveness of an oxidation

11 process may successfully be assessed by FTIR, implying a significant reduction of the

12 time devoted for analyses in comparison to other methodologies. In addition, it has been

13 shown that the results obtained by FTIR were in total agreement with those previously

14 reported using chromatographic analyses. That is, aliphatic organic compounds were

15 not easily degraded with this type of treatment, whereas phenol resulted totally

16 removed.

17 The quality and quantity of reaction intermediates that were produced during

18 the oxidative degradation of phenol were fully assessed, and the mechanisms that were

19 involved were also well observed. Thanks to receiving real-time information, this

20 procedure allowed a precise control of the effects of reagents on the treated substances,

21 which furthermore enabled optimizing the quantities of reagents required in the process.

22 This may further enable the successful optimization of the treatment combination of

23 AOPs with biological technologies, as the reaction moment where oxirecalcitrant (but

24 biodegradable) substances were mainly present in the solution was clearly identified. 


\section{Acknowledgements}

2 This research was developed in the frame of the projects "AQUAFIT4USE" (211534),

3 funded by the European Union; “AGUA Y ENERGÍA” (CTM2008-06886-C02-01),

4 and "OXIPAPEL" (CIT-310000-2008-15), both funded by the Ministry of Science and

5 Innovation of Spain. N. Merayo's participation was sponsored by a Ph.D. grant from the

6 Ministry of Economy and Competitivity of Spain.

7

8 Appendix A. Spectral characteristics of the chemical species produced along the

9 Fenton oxidation treatment of phenol

11 References

12 [1] R.J. Bigda, Consider Fenton's chemistry for wastewater treatment, Chem. Eng. Prog.

$1391(1995) 62-66$.

14 [2] S. Esplugas, J. Giménez, S. Contreras, E. Pascual, M. Rodríguez, Comparison of

15 different advanced oxidation processes for phenol degradation, Water Res. 36 (2002)

$16 \quad 1034-1042$.

17 [3] C. Comninellis, A. Kapalka, S. Malato, S.A. Parsons, I. Poulios, D. Mantzavinos,

18 Advanced oxidation processes for water treatment: advances and trends for R\&D, J.

19 Chem. Technol. Biotechnol. 83 (2008) 769-776.

20 [4] W.H. Glaze, J.W. Kang, D.H. Chapin, The Chemistry of Water-Treatment Processes

21 Involving Ozone, Hydrogen-Peroxide and Ultraviolet-Radiation, Ozone-Sci. Eng. 9

22 (1987) 335-352.

23 [5] C.P. Huang, C. Dong, Z. Tang, Advanced chemical oxidation: its present role and

24 potential future in hazardous waste treatment, Waste Manag. 13 (1993) 361-377.

25 [6] H.J.H. Fenton, Oxidation of tartaric acid in presence of iron, J. Chem. Soc. 65 
1 (1894) 899-910.

2 [7] F.J. Rivas, F.J. Beltrán, F. Carvalho, B. Acedo, O. Gimeno, Stabilized leachates:

3 sequential coagulation-flocculation plus chemical oxidation process, J. Hazard. Mater.

$4116(2004) 95-102$.

5 [8] W.Z. Tang, Physicochemical treatment of hazardous wastes, Lewis Publishers, Boca

6 Raton, FL, 2004.

7 [9] F. Harber, J.J. Weiss, The catalytic decomposition of Hydrogen Peroxide by iron

8 salts, J. Amer. Chem. Soc. 45 (1934) 338-351.

$9 \quad[10]$ J. Dzengel, J. Theurich, D.W. Bahnemann, Formation of Nitroaromatic

10 Compounds in Advanced Oxidation Processes: Photolysis versus Photocatalysis

11 Environ. Sci. Technol. 33 (1999) 294-300.

12 [11] K. Vinodgopal, Hydroxyl radical-mediated advanced oxidation processes for

13 textile dyes: a comparison of the radiolytic and sonolytic degradation of the monoazo

14 dye Acid Orange 7, J. Peller, Res. Chem. Intermed. 29, 3 (2003) 307-316.

15 [12] H. Wang, J. Wang, Electrochemical degradation of 2,4-dichlorophenol on a

16 palladium modified gas-diffusion electrode, Electrochim. Acta 53 (2008) 6402-6409.

17 [13] J. Poerschmann, U. Trommler, Pathways of advanced oxidation of phenol by

18 Fenton's reagent-Identification of oxidative coupling intermediates by extractive

19 acetylation, J. Chromatogr. A 1216 (2009) 5570-5579.

20 [14] C. Justino, A.G. Marques, K.R. Duarte, A.C. Duarte, R. Pereira, T. Rocha-Santos,

21 A.C. Freitas, Degradation of phenols in olive oil mill wastewater by biological,

22 enzymatic, and photo-Fenton oxidation, Environ Sci Pollut Res 17 (2010) 650-656.

23 [15] J. Araña, E. Tello Rendón, J.M. Doña Rodríguez, J.A. Herrera Medián, O.

24 González Díaz, J. Pérez Peña, Highly concentrated phenolic wastewater treatment by

25 the Photo-Fenton reaction, mechanism study by FTIR-ATR, Chemosphere 44 (2001) 
$1 \quad 1017-1023$.

2 [16] O. Abbas, C. Rebufa, N. Dupuy, J. Kister, FTIR-Multivariate curve resolution

3 monitoring of photo-Fenton degradation of phenolic aqueous solutions. Comparison

4 with HPLC as a reference method, Talanta 77 (2008) 200-209.

5 [17] I. Udrea, C. Bradu, Ozonation of Substituted Phenols in Aqueous Solutions over

6 CuO- $\mathrm{Al}_{2} \mathrm{O}_{3}$ Catalyst. Ozone-Sci. Eng. 25 (2003) 335-343.

7 [18] L. Carlos, D. Fabbri, A.L. Capparelli, A. Bianco Prevot, E. Pramauro, F.S. García

8 Einschlag, Intermediate distributions and primary yields of phenolic products in

9 nitrobenzene degradation by Fenton's reagent, Chemosphere 72 (2008) 952-958.

10 [19] L. Carlos, D. Fabbri, A.L. Capparelli, A. Bianco Prevot, E. Pramauro, F.S. García

11 Einschlag, Effect of simulated solar light on the autocatalytic degradation of

12 nitrobenzene using $\mathrm{Fe}^{3+}$ and hydrogen peroxide, J. Photochem. Photobiol. A: Chem. 201

13 (2009) 32-38.

14 [20] L.G. Devi, K.S.A. Raju, S.G. Kumar, Photodegradation of methyl red by advanced

15 and homogeneous photo-Fenton's processes: A comparative study and kinetic approach,

16 J. Environ. Monit. 11, 7 (2009) 1397-1404.

17 [21] B. Kayan, B. Gözmen, M. Demirel, A.M. Gizir, Degradation of acid red 97 dye in 18 aqueous medium using wet oxidation and electro-Fenton techniques, J. Hazard. Mater.

$19177(2010) 95-102$.

20 [22] L. Palmisano, M. Schiavello, A. Sclafani, G. Martra, E. Borello, S. Coluccia,

21 Photocatalysic oxidation of phenol on $\mathrm{TiO}_{2}$ powders. A Fourier transform infrared

22 study, Appl. Catal. B 3 (1994) 117-132.

23 [23] M.B. Sayed, ${ }^{1}$ H-NMR, UV-Visible, and FT-IR Spectral Analyses for the

24 Conflicting Impacts of Proton Mobility and H-Bonding Association on the Mesomeric

25 Structure in Azopyrogallol, Catechol, Resorcinol, Quinol, and Phenol Derivatives of 
1 Melamine, Ind. Eng. Chem. Res. 43 (2004) 4822-4826.

2 [24] P.Z. Araujo, C.B. Mendive, L.A. García Rodenas, P.J. Morando, A.E. Regazzoni,

3 M.A. Blesa, D. Bahnemann, FT-IR-ATR as a tool to probe photocatalytic interfaces,

4 Colloids Surf. A Physicochem. Eng. Asp. 265 (2005) 73-80.

5 [25] S. Horikoshi, T. Miura, M. Kajitani, H. Hidaka, N. Serpone, A FT-IR (DRIFT)

6 study of the influence of halogen substituents on the $\mathrm{TiO}_{2}$-assisted photooxidation of

7 phenol and $p$-halophenols under weak room light irradiance, J. Photochem. Photobiol.

8 A: Chem. 194 (2008) 189-199.

9 [26] S.A. Carr, R.B. Baird, Mineralization as a mechanism for TOC removal: study of 10 ozone/ozone-peroxide oxidation using FT-IR Water Res. 34, 16 (2000) 4036-4048.

11 [27] R.F.P. Nogueira, R.M. Alberici, M.A. Mendes, W.F. Jardim, M.N. Eberlin,

12 Photocatalytic Degradation of Phenol and Trichloroethylene: On-Line and Real-Time

13 Monitoring via Membrane Introduction Mass Spectrometry, Ind. Eng. Chem. Res. 38 14 (1999) 1754-1758.

15 [28] D. Mantzavinos, E. Psillakis, Enhancement of biodegradability of industrial 16 wastewaters by chemical oxidation pre-treatment, J. Chem. Technol. Biotechnol. 79 17 (2004) 431-454.

18 [29] I Oller, S. Malato, J.A. Sánchez-Pérez, W. Gernjak, M.I. Maldonado, L.A. Pérez-

19 Estrada, C. Pulgarín, Reduction in residual COD in biologically treated paper mill

20 effluents by means of combined ozone and ozone/UV reactor stages, Catal. Today 122 21 (2007) 150-159.

22 [30] V. Kavitha, K. Palanivelu, The role of ferrous ion in Fenton and photo-Fenton

23 processes for the degradation of phenol, Chemosphere 55 (2004) 1235-1243.

24 [31] J.A. Zazo, J.A. Casas, A.F. Mohedano, M.A. Gilarranz, J.J. Rodríguez, Chemical 25 Pathway and Kinetics of Phenol Oxidation by Fenton's Reagent, Environ. Sci. Technol. 
$139(2005) 9295-9302$.

2 [32] D. Hermosilla, M. Cortijo, C.P. Huang, The role of iron on the degradation and

3 mineralization of organic compounds using conventional Fenton and photo-Fenton

4 processes, Chem. Eng. J. 155 (2009) 637-646.

5 [33] M.S. Yalfani, S. Contreras, F. Medina, J. Sueiras, Phenol degradation by Fenton's

6 process using catalytic in situ generated hydrogen peroxide, Appl. Catal. B 89 (2009)

$7 \quad 519-526$.

8 [34] R.F.F. Pontes, J.E.F. Moraes, A. Machulek Jr., J.M. Pinto, A mechanistic kinetic

9 model for phenol degradation by the Fenton process, J. Hazard. Mater. 176 (2010) $402-$

10413.

11 [35] APHA, AWWA, WPCF (Eds.), Standard methods for the examination of water and 12 wastewater, Washington DC, 1989.

13 [36] H. Pobiner, Determination of hydroperoxides in hydrocarbon by conversion to

14 hydrogen peroxide and measurement by titanium complexing, Anal. Chem. 33 (1961)

$15 \quad 1423-1428$.

16 [37] D. Hermosilla, M. Cortijo, C.P. Huang, Optimizing the treatment of landfill

17 leachate by conventional Fenton and photo-Fenton processes, Sci. Total Environ. 407

18 (2009) 3473-3481.

19 [38] S.E. Manahan, Environmental Chemistry, CRC Press, Boca Ratón, 2010.

20 [39]V. Kavitha, K. Palanivelu, Degradation of 2-Chlorophenol by Fenton and Photo-

21 Fenton Processes-A Comparative Study, J. Environ. Sci. Heal. A 38 (2003) 1215-

221231.

23 [40] H. Kusic, N. Koprivanac, A.L. Bozic, Photo-assisted Fenton type processes for the

24 degradation of phenol: A kinetic study, Chem. Eng. J. 123 (2006) 127-137.

25 [41] M. D. Zeyaullah, A. S. Abdelkafe, W. B. Zabya, A. Ali, Biodegradation of 
1 catechols by micro-organisms - A short review, Afr. J. Biotechnol. 8 (2009) 2916-2922.

2 [42] L. Pramparo, M. E. Suárez-Ojeda, J. Pérez, J. Carrera, Kinetics of aerobic

3 biodegradation of dihydroxybenzenes by a p-nitrophenol-degrading activated sludge,

4 Bioresour. Technol. 110 (2012) 57-62.

5 [43] D. Hermosilla, N. Merayo, R. Ordóñez, A. Blanco, Optimization of conventional

6 Fenton and ultraviolet-assisted oxidation processes for the treatment of reverse osmosis

7 retentate from a paper mill, Waste Manage. 32 (2012) 1236-1243.

8 [44] S. Kim, A. Vogelpohl, Degradation of Organic Pollutants by the Photo-Fenton-

9 Process, Chem. Eng. Technol. 21 (1998) 187-191.

10 [45] M.I. Stefan, J.R. Bolton, Mechanism of the Degradation of 1,4-Dioxane in Dilute

11 Aqueous Solution Using the UV/Hydrogen Peroxide Process, Environ. Sci. Technol. 32

12 (1998) 1588-1595.

13 [46] I.R. Moraes, F.C. Nart, Sulfate ions adsorbed on Au(hkl) electrodes: in situ

14 vibrational spectroscopy, J. Electroanal. Chem. 461 (1999) 110-120.

15 [47] Y.M. Jung, Characterization of pH-Dependent IR Spectra of Oxalic Acid:

16 Comparison of Self-Modeling Curve Resolution Analysis with Calculation of IR

17 Frequencies, Bull. Korean Chem. Soc. 24, 9 (2003) 1410-1412.

18 [48] B. Jin, P. Liu, Y. Wang, Z. Zhang, Y. Tian, J. Yang, S. Zhang, F. Cheng, Rapid-

19 Scan Time-Resolved FT-IR Spectroelectrochemistry Studies on the Electrochemical

20 Redox Process, J. Phys. Chem. B 111 (2007) 1517-1522.

21 [49] J. Araña, J.M. Doña Rodríguez, O. González Díaz, J.A. Herrera Melián, C.

22 Fernández Rodríguez, J. Pérez Peña, The effect of acetic acid on the photocatalytic

23 degradation of catechol and resorcinol, Appl. Catal. A Gen. 299 (2006) 274-284.

24 [50] H. Gulley-Stahl, P.A. Hogan II, W.L. Schmidt, S.J. Wall, A. Buhrlage, A.A.

25 Bullen, Surface Complexation of Catechol to Metal Oxides: An ATR-FTIR, 
1 Adsorption, and Dissolution Study, Environ. Sci. Technol. 44 (2010) 4116-4121.

2 [51] F.C. Nart, T. Iwasita, M. Weber, Sulfate adsorption on well-define $\operatorname{Pt}(100)$

3 electrodes, Electrochim. Acta 39, 13 (1994) 2093-2096.

4 [52] R.T.S. Muthu Lakshmi, M.K. Vyas, A.S. Brar, I.K. Varma, Synthesis and

5 characterization of sulphonated PEES copolymers by NMR spectroscopy, Eur. Polym.

6 J. $42(2006)$ 1423-1432.

7 [53] M.M. Hasani-Sadrabadi, S.H. Emami, H. Moaddel, Preparation and

8 characterization of nanocomposite membranes made of poly(2,6-dimethyl-1,4-

9 phenylene oxide) and montmorillonite for direct methanol fuel cells, J. Power Sources

$10183(2008) 551-556$. 
Table A.1. Characteristic wavenumber peaks of relevant organic compounds that are produced during the Fenton oxidation of phenol, oxalic acid and acetic acid.

\begin{tabular}{|c|c|c|c|c|c|c|c|c|c|}
\hline \multicolumn{2}{|c|}{ Wavenumber $\left(\mathrm{cm}^{-1}\right)$} & \multirow[b]{2}{*}{ Resorcinol } & \multirow[b]{2}{*}{ Catechol } & \multirow[b]{2}{*}{ Benzoquinone } & \multirow[b]{2}{*}{ Oxalic acid } & \multirow[b]{2}{*}{ Acetic acid } & \multirow[b]{2}{*}{ Formic acid } & \multirow[b]{2}{*}{$\mathrm{FeSO}_{4} \cdot 7 \mathrm{H}_{2} \mathrm{O}$} & \multirow[b]{2}{*}{ References } \\
\hline Phenol & Hydroquinone & & & & & & & & \\
\hline & & & & & 1738 & & & & {$[47]$} \\
\hline & & & & & & 1707 & & & [16] \\
\hline & & & & 1638 & & & & & {$[48]$} \\
\hline & & & & & 1621 & & & & [47] \\
\hline \multirow[t]{2}{*}{1595} & & 1604 & 1604 & 1599 & & & & & {$[16,22,25,49-51]$} \\
\hline & & & & & 1574 (anion) & & & & {$[47]$} \\
\hline 1501 & & & & & & & & & {$[16,22,25]$} \\
\hline \multirow[t]{4}{*}{1475} & 1472 & & & & & & & & {$[16,22,25]$} \\
\hline & & & & & & 1410 & & & [16] \\
\hline & & 1381 & & & & & & & [49] \\
\hline & & & 1379 & & & & & & {$[49-50]$} \\
\hline \multirow[t]{4}{*}{1372} & & & & & & & & & {$[16,22,25]$} \\
\hline & 1367 & & & & & & & & [16] \\
\hline & & & & & 1310 (anion) & & & & [47] \\
\hline & & & & & & 1292 & & & [16] \\
\hline \multirow[t]{4}{*}{1235} & & & & & 1232 & & & & {$[16,22,25,47]$} \\
\hline & & & & & 1226 & & & & {$[35]$} \\
\hline & & & 1200 & & & & & & {$[16,49,50]$} \\
\hline & & & & & & & & 1089 & [51-53] \\
\hline 809 & & & & & & & & & {$[16,22,25]$} \\
\hline 752 & & & & & & & & & {$[16,22,25]$} \\
\hline
\end{tabular}




\section{Figure captions}

Figure A.1. FTIR spectra of main inorganic and aliphatic compounds that are used and may be produced in the oxidation of phenol by the Fenton process.

Figure A.2. FTIR spectra of main aromatic chemicals that may be produced in the oxidation of phenol by the Fenton process.

Figure 1. Evolution of FTIR-spectra along a continuous hydrogen peroxide addition in the Fenton oxidation of phenol. The presence evolution of carboxylic acids is highlighted, and a turned around graph is also shown in small detail. (Reaction conditions: room temperature $\left(\approx 20-25^{\circ} \mathrm{C}\right) ; \mathrm{pH}=2.8 \pm 0.2 ; 16 \mathrm{mmol}$ phenol; $\left[\mathrm{H}_{2} \mathrm{O}_{2}\right] /$ $\left.\mathrm{COD}_{0}=2.15 ;\left[\mathrm{H}_{2} \mathrm{O}_{2}\right] /\left[\mathrm{Fe}^{2+}\right]=37.5\right)$.

Figure 2. Absorbance profiles for main by-products, and evolution of the MOC along a continuous hydrogen peroxide addition in the Fenton oxidation of phenol (Reaction conditions: room temperature $\left(\approx 20-25^{\circ} \mathrm{C}\right) ; \mathrm{pH}=2.8 \pm 0.2 ; 16 \mathrm{mmol}$ phenol; $\left[\mathrm{H}_{2} \mathrm{O}_{2}\right] /$ $\left.\mathrm{COD}_{0}=2.15 ;\left[\mathrm{H}_{2} \mathrm{O}_{2}\right] /\left[\mathrm{Fe}^{2+}\right]=37.5\right)$.

Figure 3. Smooth absorbance concentration profiles of phenol, and evolution of the removal of the COD along the Fenton oxidation of phenol considering all its three typical detection wavenumbers $\left(752,809\right.$, and $\left.1501 \mathrm{~cm}^{-1}\right)$. Note: $1=$ addition of reagents; $2=$ progressive removal of phenol; $3=$ after the total removal of phenol. 
Figure 4. Concentration profiles of acetic acid, formic acid and organic-iron complexes during the degradation of acetic acid by Fenton's reagent. (Reaction conditions: 12 mmol acetic acid, $\left.\mathrm{pH}=2.8 \pm 0.2,\left[\mathrm{H}_{2} \mathrm{O}_{2}\right] /\left[\mathrm{Fe}^{2+}\right]=37.5\right)$.

Figure 5. FTIR spectra resulting for deionised water, phenol, acetic acid, and oxalic acid with and without the presence of ferric chloride trihydrate.

Figure 6. Evolution of FTIR spectra in the span ranging from 2000 to $800 \mathrm{~cm}^{-1}$ (A), and concentration profiles of oxalic acid and oxalate (B) along the Fenton oxidation of oxalic acid. (Reaction conditions: 14 mmol oxalic acid, $\mathrm{pH}=2.8 \pm 0.2,\left[\mathrm{H}_{2} \mathrm{O}_{2}\right] /\left[\mathrm{Fe}^{2+}\right]=$ $37.5)$.

Figure 7. Absorbance concentration profiles for characteristic wavenumber values of oxalic acid (1733 and $\left.1232 \mathrm{~cm}^{-1}\right)$ and its anion (1571 and $\left.1310 \mathrm{~cm}^{-1}\right)$ considering the following $\mathrm{pH}$ turns: (A) natural $\mathrm{pH}$ of an oxalic acid solution, (B) $40 \% \mathrm{NaOH}$ addition to increase $\mathrm{pH}$, and $(\mathrm{C}) \mathrm{pH}$ values at which oxalic forms are stabilized $(>9.0)$. 


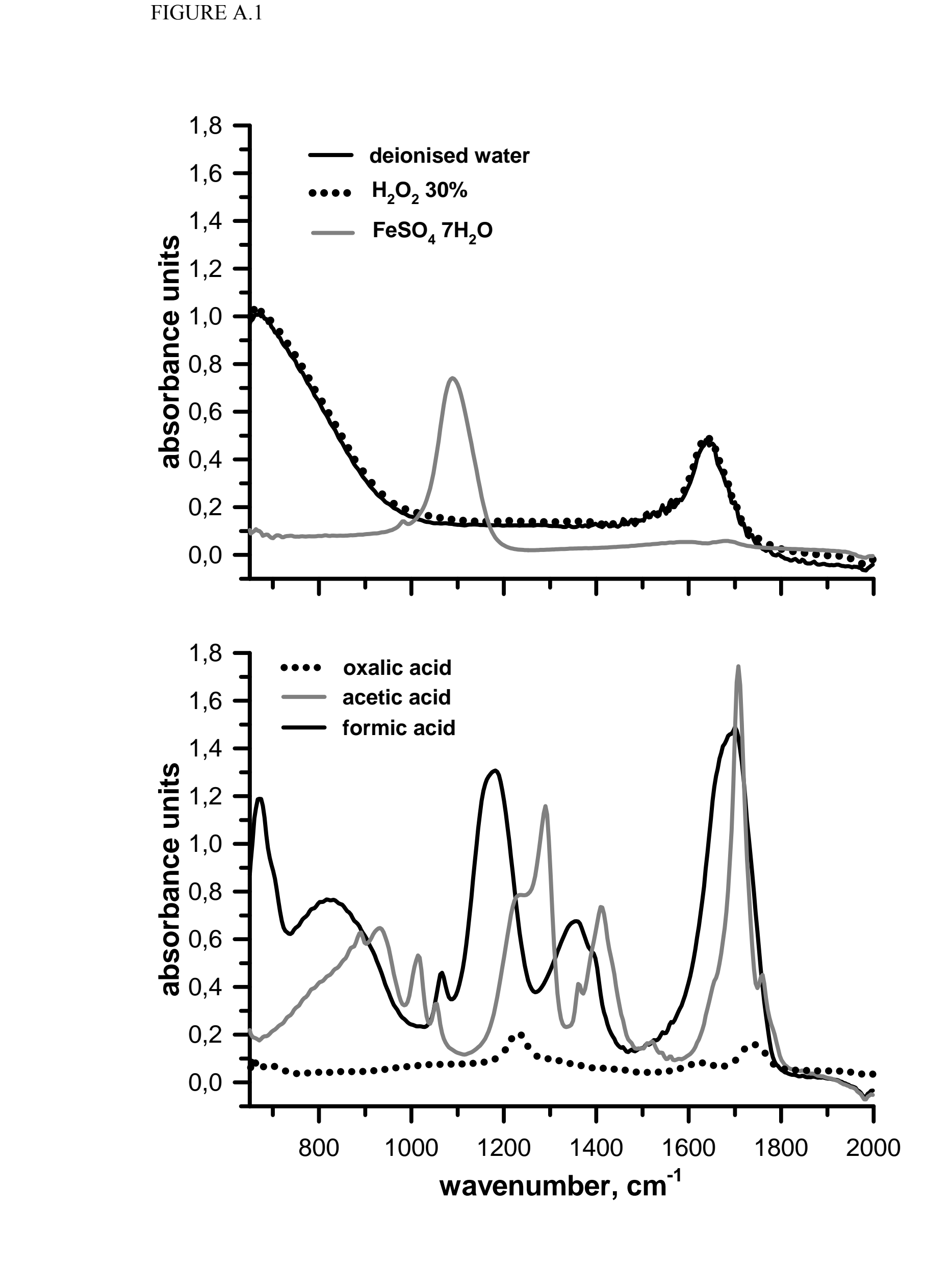


FIGURE A.2.
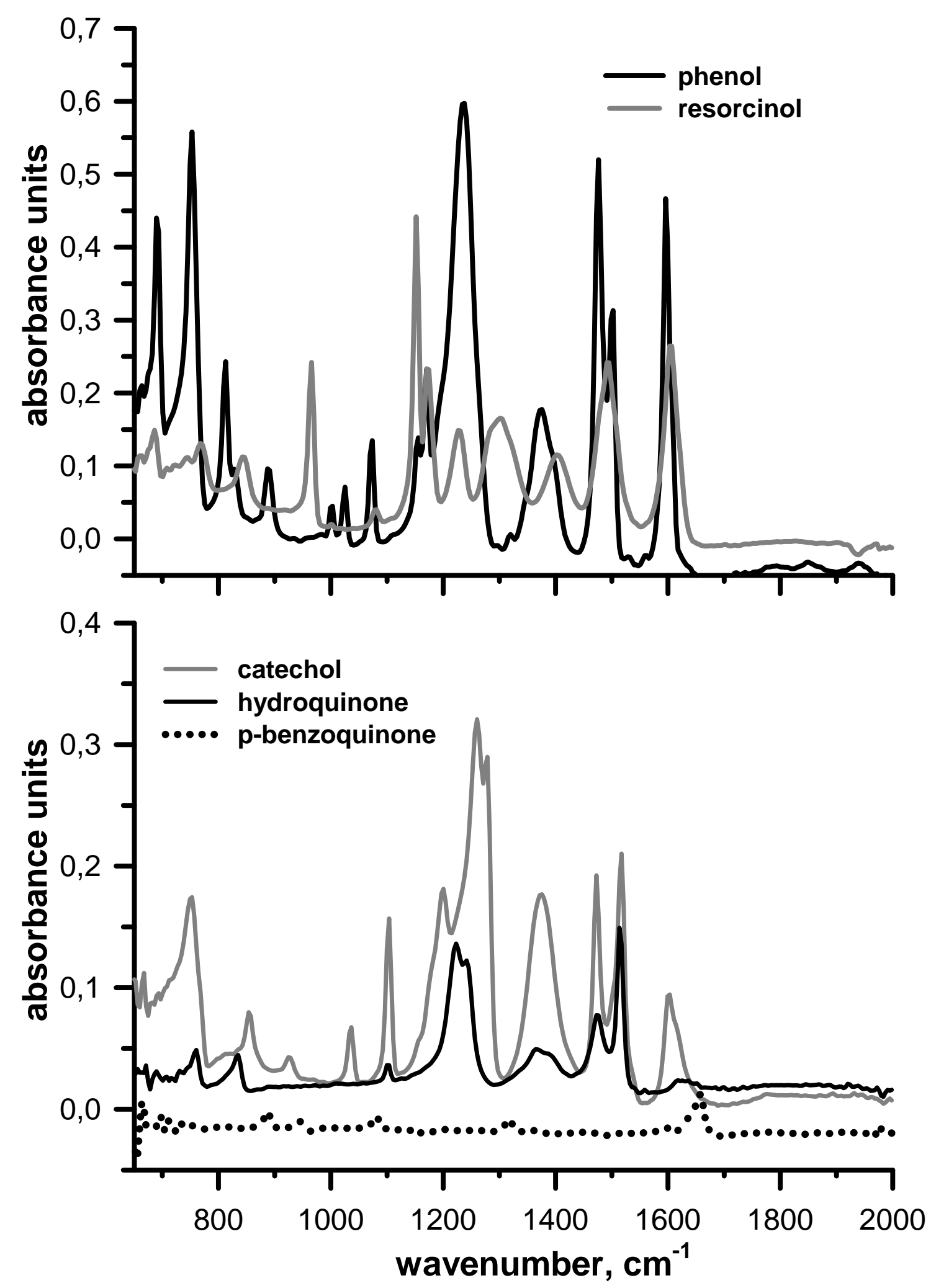
FIGURE 1

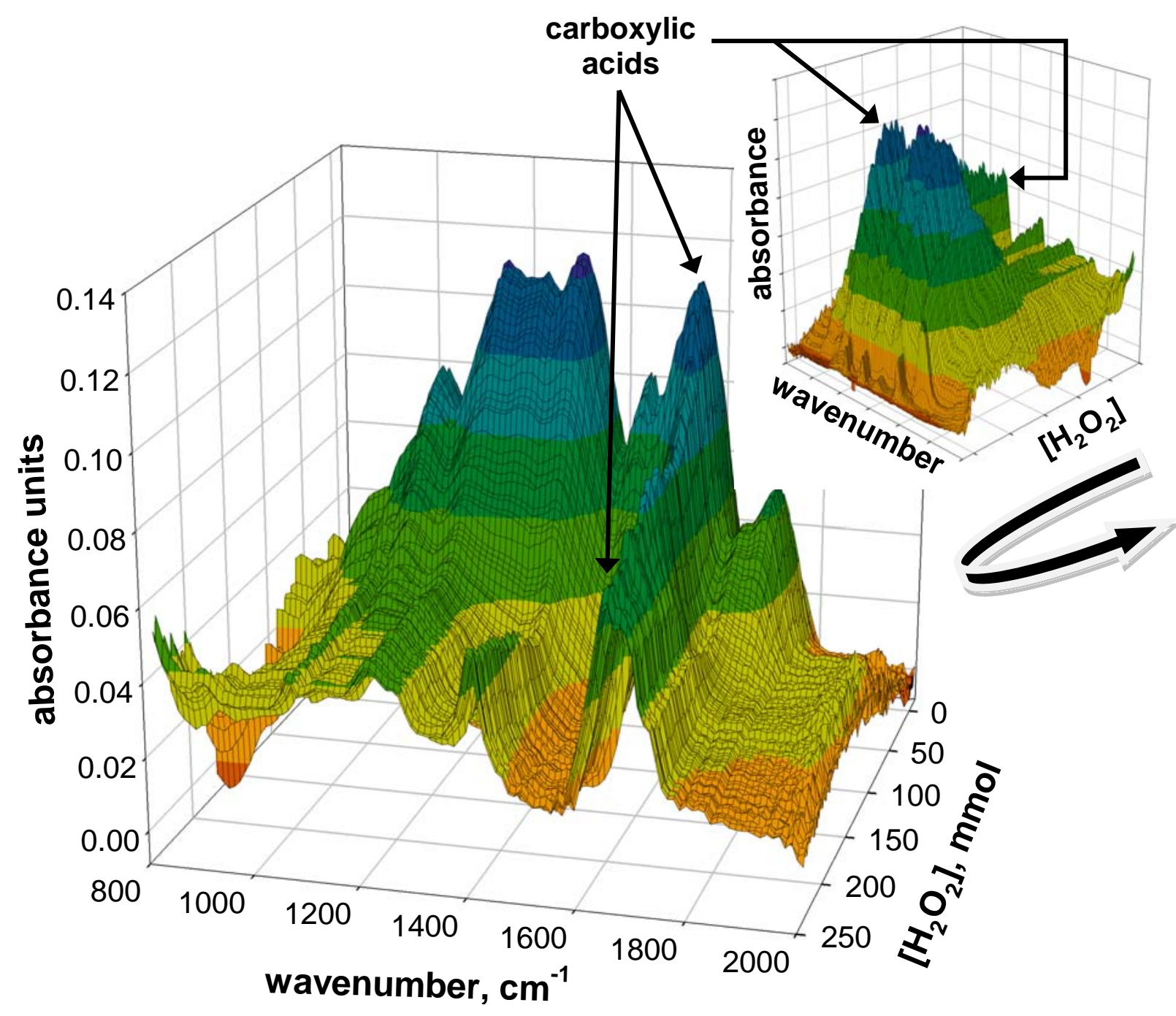


FIGURE 2

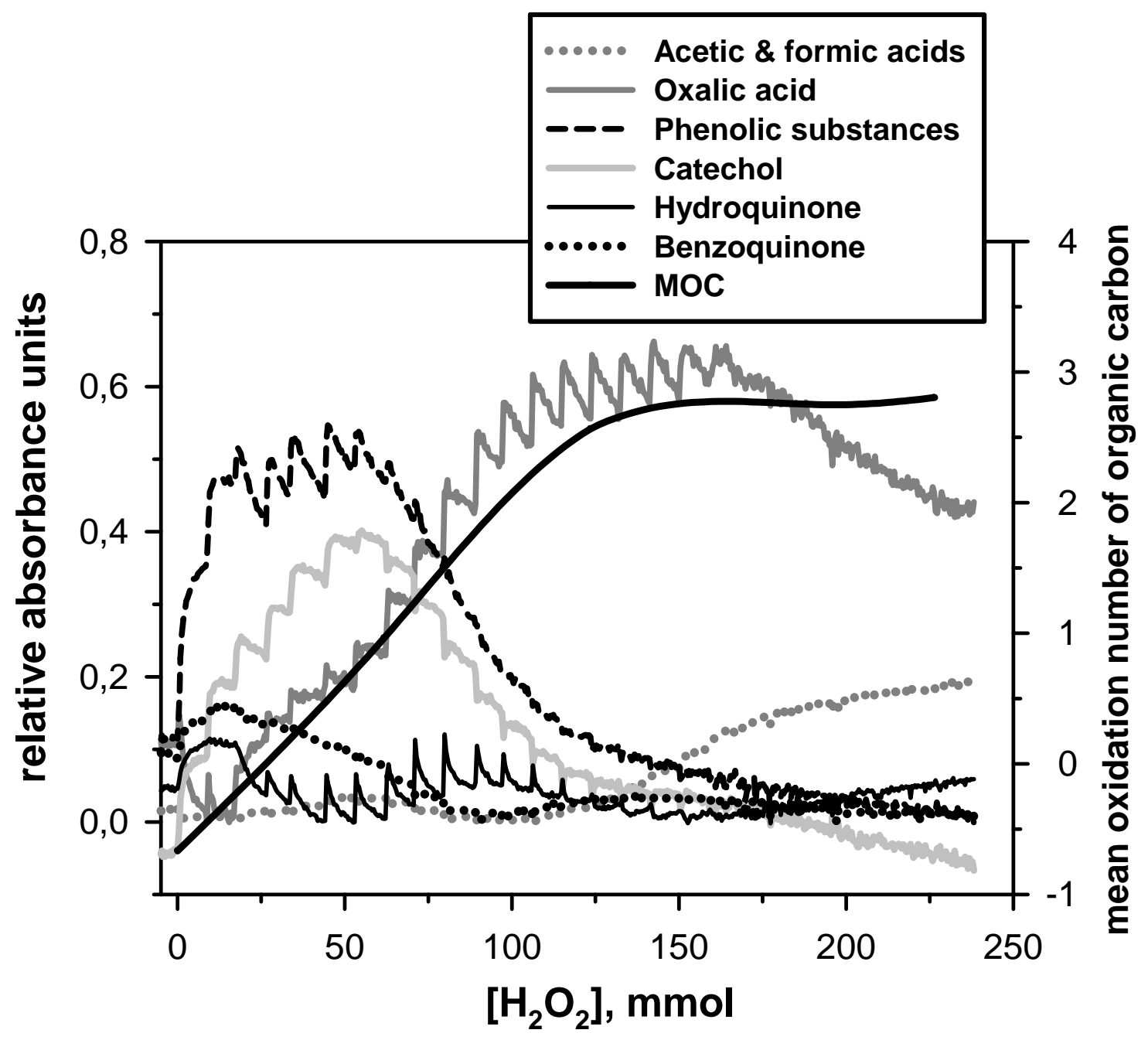


FIGURE 3

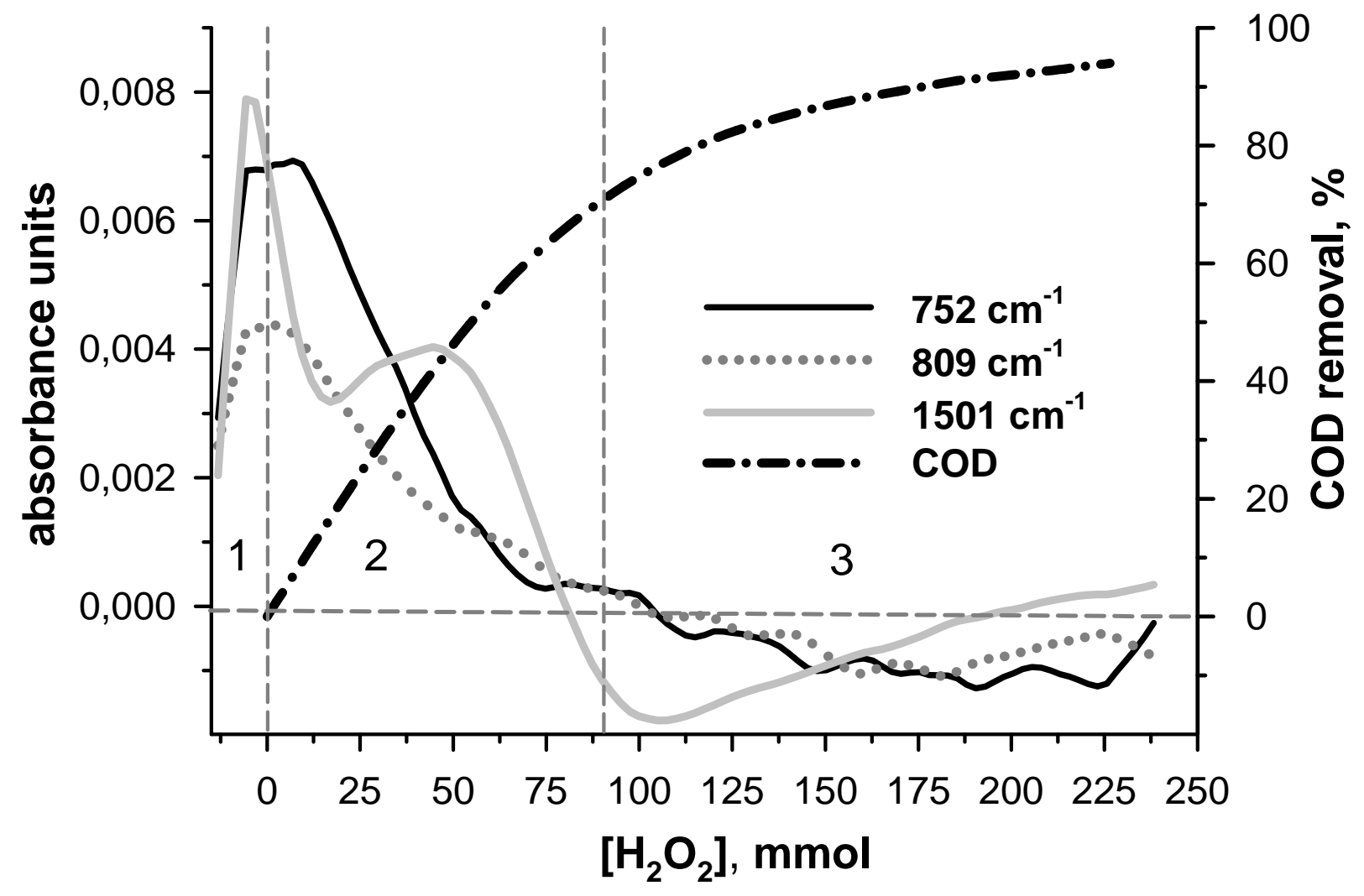


FIGURE 4

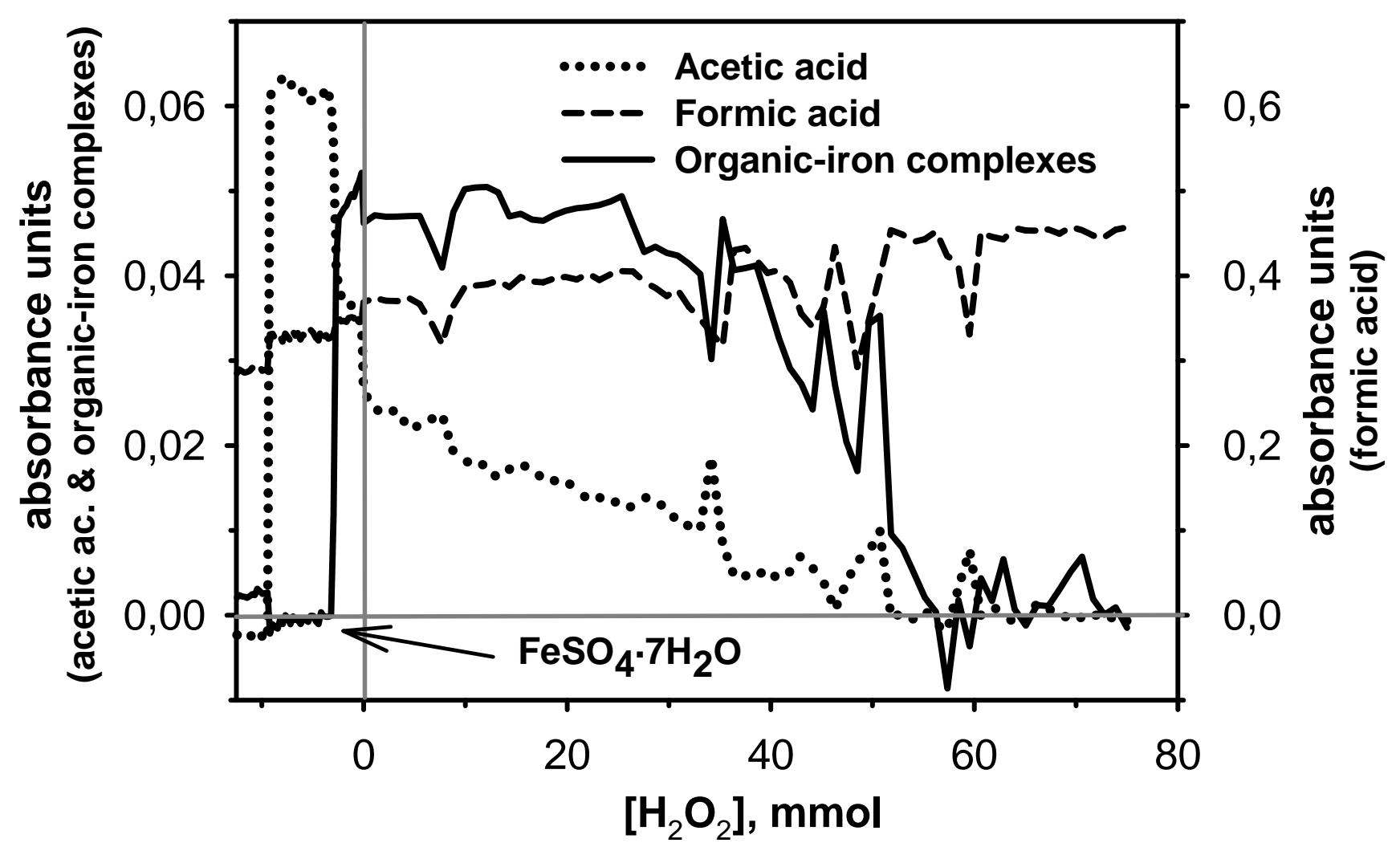


FIGURE 5

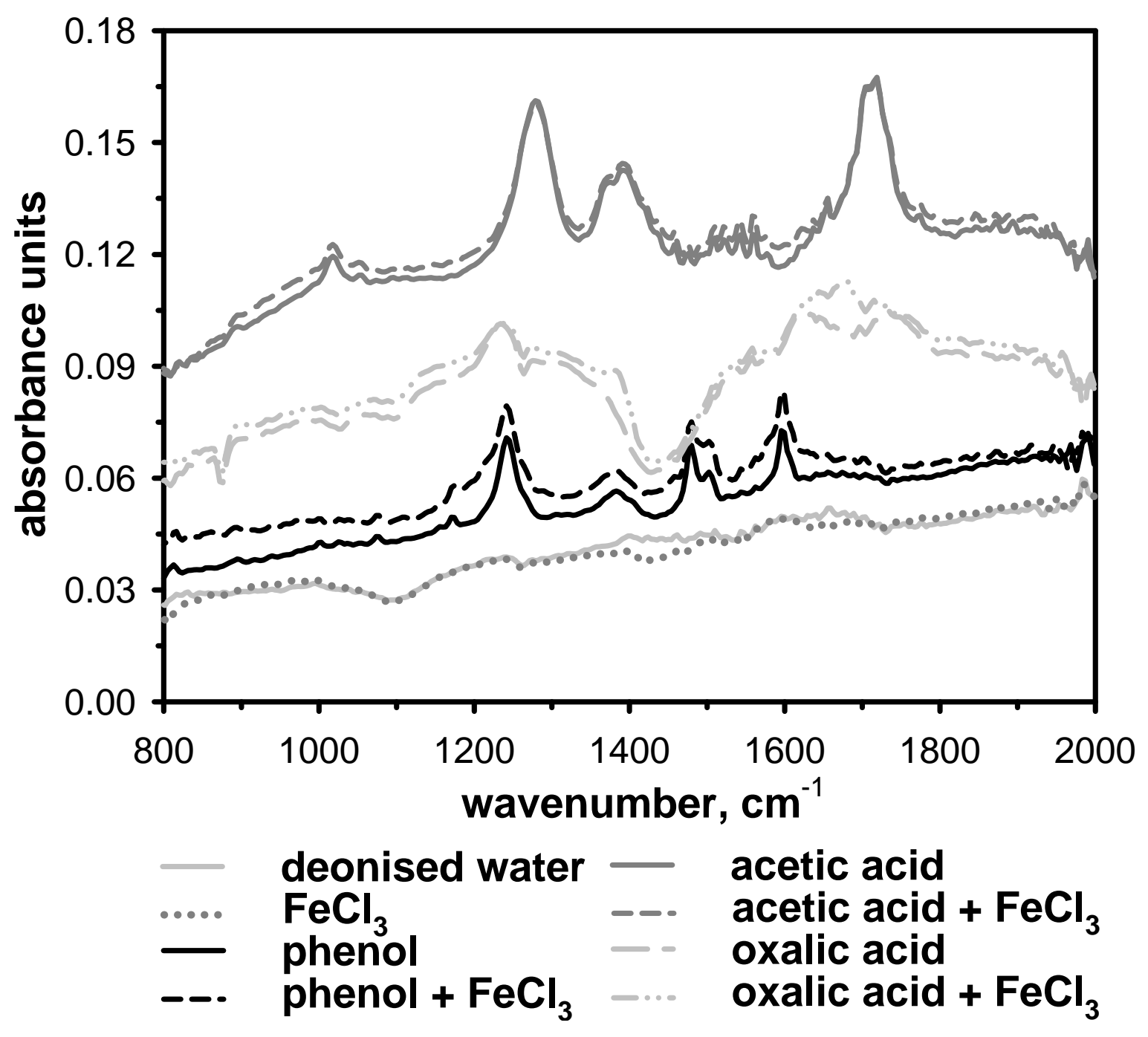


FIGURE 6
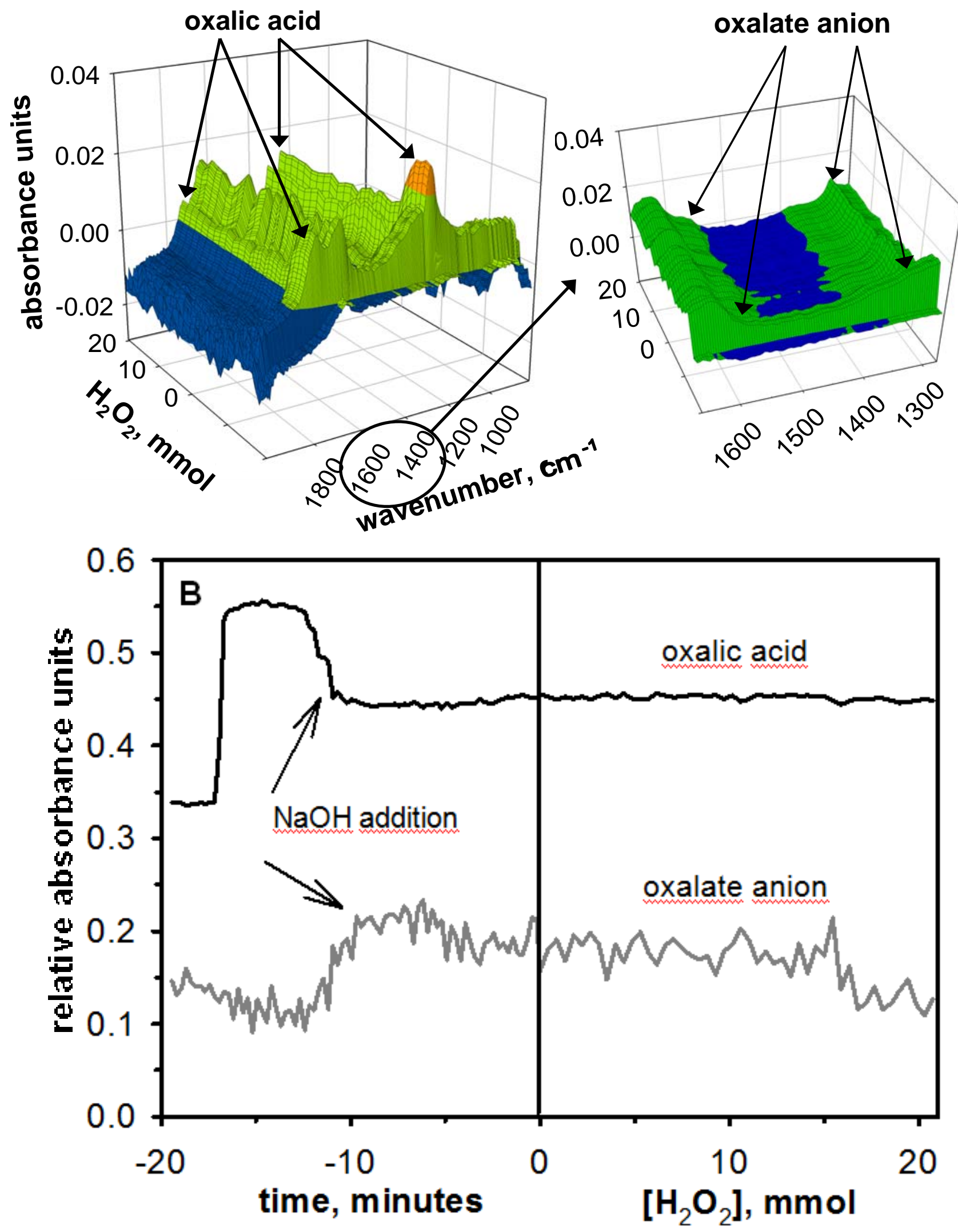
FIGURE 7

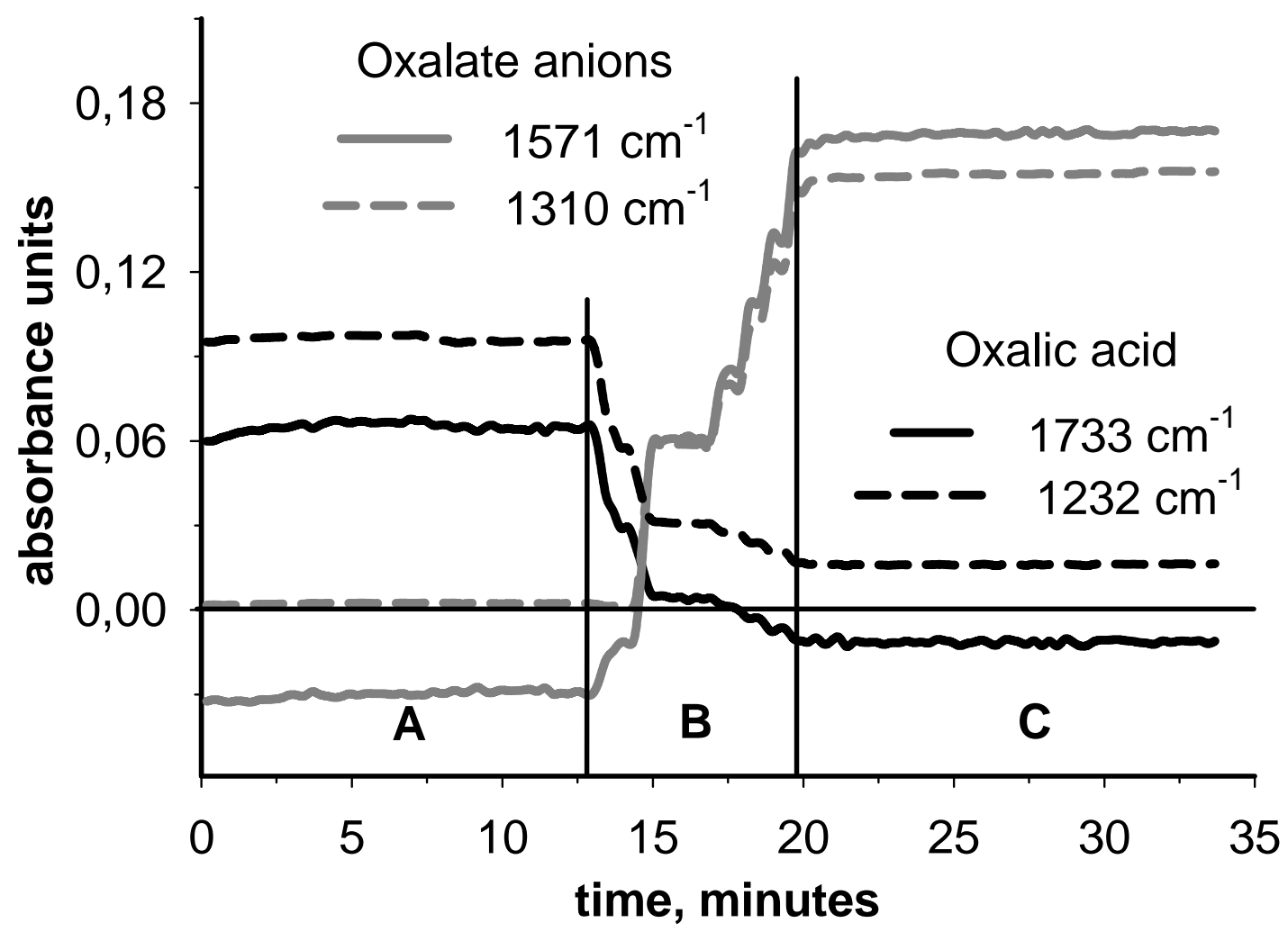

